\title{
Reasoning about Knowledge and Strategies: Epistemic Strategy Logic
}

\author{
Francesco Belardinelli \\ Laboratoire IBISC - Unversité d'Evry \\ belardinelli@ibisc.fr
}

\begin{abstract}
In this paper we introduce Epistemic Strategy Logic (ESL), an extension of Strategy Logic with modal operators for individual knowledge. This enhanced framework allows us to represent explicitly and to reason about the knowledge agents have of their own and other agents' strategies. We provide a semantics to ESL in terms of epistemic concurrent game models, and consider the corresponding model checking problem. We show that the complexity of model checking ESL is not worse than (non-epistemic) Strategy Logic.
\end{abstract}

\section{Introduction}

Formal languages to represent and reason about strategies and coalitions are a thriving area of research in Artificial Intelligence and multi-agent system [4, 8, 19]. Recently, a wealth of multi-modal logics have appeared, which allow to formalise complex strategic abilities and behaviours of individual agents and groups [2, 5]. In parallel to these developments, in knowledge representation there is a well-established tradition of extending logics for reactive systems with epistemic operators to reason about the knowledge agents have of systems evolution. These investigations began in the ' 80 s with contributions on combinations of linear- and branching-time temporal logics with multi-agent epistemic languages [9, 10, 6]. Along this line of research, [11] introduced alternating-time temporal epistemic logic (ATEL), an extension of ATL with modalities for individual knowledge. The various flavours of logics of time and knowledge have been successfully applied to the specification of distributed and multi-agent systems in domains as diverse as security protocols, UAVs, web services, and e-commerce, as well as to verification by model checking [7, 16].

In this paper we take inspiration from the works above and pursue further this line of research by introducing Epistemic Strategy Logic, an extension of Strategy Logic (SL) [5, 17] that allows agents to reason about their strategic abilities. The extension here proposed is naive in the sense that it suffers many of the shortcomings of its relative ATEL [12]. Nonetheless, we reckon that it constitutes an excellent starting point to analyse the interaction of knowledge and strategic abilities in a language, such as SL, that explicitly allow for quantification on strategies.

Related Work. This paper builds on previous contributions on Strategy Logic. SL has been introduced in [5] for two-player concurrent game structures (CGS). In [17] the semantics has been extended to a multi-player setting. Also, [17] introduced bind operators for strategies in the syntax. In the present contribution we consider multi-agent CGS in line with [17]. However, we adopt an agent-based perspective and consider agents with possibly different actions and protocols [6]. Also, our language do not include bind operators to avoid the formal machinery associated with these operators. We leave such an extension for future and more comprehensive work. Finally, the model checking results in Section 4 are inspired by and use techniques from [17].

F. Mogavero, A. Murano, and M.Y. Vardi (Eds.): 2nd Workshop on Strategic Reasoning 2014 (SR14)

EPTCS 146, 2014, pp. $27-33$ doi 10.4204/EPTCS.146.4 (c) F. Belardinelli

This work is licensed under the Creative Commons Attribution License. 
Even though to our knowledge no epistemic extension of SL has been proposed yet, the interaction between knowledge and strategic reasoning has been studied extensively, especially in the context of alternating-time temporal logic. An extension of ATL with knowledge operators, called ATEL, was put forward in [11], and immediately imperfect information variants of this logic were considered in [14], which introduces alternating-time temporal observational logic (ATOL) and ATEL-R*, as well as uniform strategies. Notice that [14] also analyses the distinction between de re and de dicto knowledge of strategies; this distinction will also be considered later on in the context of Epistemic Strategy Logic. Further, [13] enriches ATL with a constructive notion of knowledge. As regards (non-epistemic) ATL, more elaborate notions of strategy have been considered. In [1] commitment in strategies has been analysed; while [15] introduced a notion of "feasible" strategy. In future work it might be worth exploring to what extent the theoretical results available for the various flavours of ATEL transfer to ESL.

Scheme of the paper. In Section 2 we introduce the epistemic concurrent game models (ECGM), which are used in Section 3 to provide a semantics to Epistemic Strategy Logic (ESL). In Section 4 we consider the model checking problem for this setting and state the corresponding complexity results. Finally, in Section 5 we discuss the results and point to future research. For reasons of space, all proofs are omitted. An extended version of this paper with complete proofs is available [3].

\section{Epistemic Concurrent Game Models}

In this section we present the epistemic concurrent game models (ECGM), an extension of concurrent game structures [2, 11], starting with the notion of agent.

Definition 1 (Agent) An agent is a tuple $i=\left\langle L_{i}, A c t_{i}, P r_{i}\right\rangle$ such that (i) $L_{i}$ is the set of local states $l_{i}, l_{i}^{\prime}, \ldots$; (ii) Act $t_{i}$ is the finite set of actions $\sigma_{i}, \sigma_{i}^{\prime}, \ldots$; and (iii) $P r_{i}: L_{i} \mapsto 2^{A c t_{i}}$ is the protocol function.

Intuitively, each agent $i$ is situated in some local state $l_{i} \in L_{i}$, representing her local information, and performs the actions in $A c t_{i}$ according to the protocol function $\operatorname{Pr}_{i}$ [6]. Differently from [17], we assume that agents have possibly different actions and protocols. To formally describe the interactions between agents, we introduce their synchronous composition. Given a set $A P$ of atomic propositions and a set $A g=\left\{i_{0}, \ldots, i_{n}\right\}$ of agents, we define the set $L$ of global states $s, s^{\prime}, \ldots$ (resp. the set Act of joint actions $\left.\sigma, \sigma^{\prime}, \ldots\right)$ as the cartesian product $L_{0} \times \ldots \times L_{n}$ (resp. $\left.A c t_{0} \times \ldots \times A c t_{n}\right)$. In what follows we denote the $j$ th component of a tuple $t$ as $t_{j}$ or, equivalently, as $t(j)$.

Definition 2 (ECGM) Given a set $A g=\left\{i_{0}, \ldots, i_{n}\right\}$ of agents $i=\left\langle L_{i}, A c t_{i}, P r_{i}\right\rangle$, an epistemic concurrent game model is a tuple $\mathscr{P}=\left\langle A g, s_{0}, \tau, \pi\right\rangle$ such that (i) $s_{0} \in L$ is the initial global state; (ii) $\tau: L \times A c t \mapsto L$ is the global transition function, where $\tau(s, \sigma)$ is defined iff $\sigma_{i} \in \operatorname{Pr}_{i}\left(l_{i}\right)$ for every $i \in A g$; and (iii) $\pi$ : $A P \mapsto 2^{L}$ is the interpretation function for atomic propositions in $A P$.

The transition function $\tau$ describes the evolution of the ECGM from the initial state $s_{0}$. We now introduce some notation that will be used in the rest of the paper. The transition relation $\rightarrow$ on global states is defined as $s \rightarrow s^{\prime}$ iff there exists $\sigma \in A c t$ s.t. $\tau(s, \sigma)=s^{\prime}$. A run $\lambda$ from a state $s$, or $s$-run, is an infinite sequence $s^{0} \rightarrow s^{1} \rightarrow \ldots$, where $s^{0}=s$. For $n, m \in \mathbb{N}$, with $n \leq m$, we define $\lambda(n)=s^{n}$ and $\lambda[n, m]=s^{n}, s^{n+1}, \ldots, s^{m}$. A state $s^{\prime}$ is reachable from $s$ if there exists an $s$-run $\lambda$ s.t. $\lambda(i)=s^{\prime}$ for some $i \geq 0$. We define $S$ as the set of states reachable from the initial state $s_{0}$. Further, let $\sharp$ be a placeholder for arbitrary individual actions. Given a subset $A \subseteq A g$ of agents, an $A$-action $\sigma_{A}$ is an $|A g|-$ tuple s.t. $(i) \sigma_{A}(i) \in A c t_{i}$ for $i \in A$, and (ii) $\sigma_{A}(j)=\sharp$ for $j \notin A$. Then, $A c t_{A}$ is the set of all $A$-actions and $D_{A}(s)=\left\{\sigma_{A} \in A c t_{A} \mid\right.$ for every $\left.i \in A, \sigma_{i} \in P r_{i}\left(l_{i}\right)\right\}$ is the set of all $A$-actions enabled at $s=\left\langle l_{0}, \ldots, l_{n}\right\rangle$. A joint action $\sigma$ extends an $A$-action $\sigma_{A}$, or $\sigma_{A} \sqsubseteq \sigma$, iff $\sigma_{A}(i)=\sigma(i)$ for all $i \in A$. The outcome out $\left(s, \sigma_{A}\right)$ 
of action $\sigma_{A}$ at state $s$ is the set of all states $s^{\prime}$ s.t. there exists a joint action $\sigma \sqsupseteq \sigma_{A}$ and $\tau(s, \sigma)=s^{\prime}$. Finally, two global states $s=\left\langle l_{0}, \ldots, l_{n}\right\rangle$ and $s^{\prime}=\left\langle l_{0}^{\prime}, \ldots, l_{n}^{\prime}\right\rangle$ are indistinguishable for agent $i$, or $s \sim_{i} s^{\prime}$, iff $l_{i}=l_{i}^{\prime}[6]$.

\section{Epistemic Strategy Logic}

We now introduce Epistemic Strategy Logic as a specification language for ECGM. Hereafter we consider a set $\operatorname{Var}_{i}$ of strategy variables $x_{i}, x_{i}^{\prime}, \ldots$, for every agent $i \in A g$.

Definition 3 (ESL) For $p \in A P, i \in A g$ and $x_{i} \in$ Var $_{i}$, the ESL formulas $\phi$ are defined in BNF as follows:

$$
\phi \quad::=p|\neg \phi| \phi \rightarrow \phi|X \phi| \phi U \phi\left|K_{i} \phi\right| \exists x_{i} \phi
$$

The language ESL is an extension of the Strategy Logic in [5] to a multi-agent setting, including an epistemic operator $K_{i}$ for each $i \in A g$. Alternatively, ESL can be seen as the epistemic extension of the Strategy Logic in [17], minus the bind operator. We do not consider bind operators in ESL for ease of presentation. The ESL formula $\exists x_{i} \phi$ is read as "agent $i$ has some strategy to achieve $\phi$ ". The interpretation of LTL operators $X$ and $U$ is standard. The epistemic formula $K_{i} \phi$ intuitively means that "agent $i$ knows $\phi$ ". The other propositional connectives and LTL operators, as well as the strategy operator $\forall$, can be defined as standard. Also, notice that we can introduce the nested-goal fragment ESL[NG], the boolean-goal fragment ESL[BG], and the one-goal fragment ESL[1G] in analogy to SL [17]. Further, the free variables $f r(\phi) \subseteq A g$ of an ESL formula $\phi$ are inductively defined as follows:

$$
\begin{array}{ll}
f r(p) & =\emptyset \\
f r(\neg \phi)=f r\left(K_{i} \phi\right) & =f r(\phi) \\
f r\left(\phi \rightarrow \phi^{\prime}\right) & =f r(\phi) \cup f r\left(\phi^{\prime}\right) \\
f r(X \phi)=f r\left(\phi U \phi^{\prime}\right) & =A g \\
f r\left(\exists x_{i} \phi\right) & =f r(\phi) \backslash\{i\}
\end{array}
$$

A sentence is a formula $\phi$ with $f r(\phi)=\emptyset$, and the set $b n d(\phi)$ of bound variables is defined as $A g \backslash f r(\phi)$.

To provide a semantics to ESL formulas in terms of ECGM, we introduce the notion of strategy.

Definition 4 (Strategy) Let $\gamma$ be an ordinal s.t. $1 \leq \gamma \leq \omega$ and $A \subseteq A g$ a set of agents. $A \gamma$-recall $A$-strategy is a function $F_{A}[\gamma]: \bigcup_{1 \leq n<1+\gamma} S^{n} \mapsto \bigcup_{s \in S} D_{A}(s)$ s.t. $F_{A}[\gamma](\kappa) \in D_{A}($ last $(\kappa))$ for every $\kappa \in$ $\bigcup_{1 \leq n<1+\gamma} S^{n}$, where $1+\gamma=\gamma$ for $\gamma=\omega$ and last $(\kappa)$ is the last element of $\kappa$.

Hence, a $\gamma$-recall $A$-strategy returns an enabled $A$-action for every sequence $\kappa \in \bigcup_{1 \leq n<1+\gamma} S^{n}$ of states of length at most $\gamma$. Notice that for $A=\{i\}, F_{A}[\gamma]$ can be seen as a function from $\bigcup_{1 \leq n<1+\gamma} S^{n}$ to $A c t_{i}$ s.t. $F_{A}[\gamma](\kappa) \in \operatorname{Pr}_{i}($ last $(\kappa))$ for $\kappa \in \bigcup_{1 \leq n<1+\gamma} S^{n}$. In what follows we write $F_{i}[\gamma]$ for $F_{\{i\}}[\gamma]$. Then, for $A=\left\{i_{0}, \ldots, i_{m}\right\} \subseteq A g, F_{A}[\gamma]$ is equal to $F_{i_{0}}[\gamma] \times \ldots \times F_{i_{m}}[\gamma]$, where for every $\kappa \in \bigcup_{1 \leq n<1+\gamma} S^{n}$, $\left(F_{i_{0}}[\gamma] \times \ldots \times F_{i_{m}}[\gamma]\right)(\kappa)$ is defined as the set of actions $\sigma \in \bigcup_{s \in S} D_{A}(s)$ s.t. $\sigma_{i}=F_{i}[\gamma](\kappa)$ if $i \in A, \sigma_{i}=\sharp$ otherwise. Therefore, a group strategy is the composition of its members' strategies. Further, the outcome of strategy $F_{A}[\gamma]$ at state $s$, or $\operatorname{out}\left(s, F_{A}[\gamma]\right)$, is the set of all $s$-runs $\lambda$ s.t. $\lambda(i+1) \in \operatorname{out}(\lambda(i), F[\gamma](\lambda[j, i]))$ for all $i \geq 0$ and $j=\max (i-\gamma+1,0)$. Depending on $\gamma$ we can define positional strategies, strategies with perfect recall, etc. [8]. However, these different choices do not affect the following results, so we assume that $\gamma$ is fixed and omit it. Moreover, by Def. 4 it is apparent that agents have perfect information, as their strategies are determined by global states [4]; we leave contexts of imperfect information for future research.

Now let $\chi$ be an assignment that maps each agent $i \in A g$ to an $i$-strategy $F_{i}$. For $A g=\left\{i_{0}, \ldots, i_{n}\right\}$, we denote $\chi\left(i_{0}\right) \times \ldots \times \chi\left(i_{n}\right)$ as $F^{\chi}$, that is, the $A g$-strategy s.t. for every $\kappa \in \cup_{1 \leq n<1+\gamma} S^{n}, F^{\chi}(\kappa)=\sigma \in$ 
$\bigcup_{s \in S} D_{A g}(s)$ iff $\sigma_{i}=\chi(i)(\kappa)$ for every $i \in A g$. Since $\mid$ out $\left(s, F^{\chi}\right) \mid=1$, we simply write $\lambda=$ out $\left(s, F^{\chi}\right)$. Also, $\chi_{F_{i}}^{i}$ denotes the assignment s.t. ( $\left.i\right)$ for all agents $j$ different from $i, \chi_{F_{i}}^{i}(j)=\chi(j)$, and $(i i) \chi_{F_{i}}^{i}(i)=F_{i}$.

Definition 5 (Semantics of ESL) We define whether an ECGM $\mathscr{P}$ satisfies a formula $\varphi$ at state $s$ according to assignment $\chi$, or $(\mathscr{P}, s, \chi)=\varphi$, as follows (clauses for propositional connectives are straightforward and thus omitted):

$$
\begin{array}{lll}
(\mathscr{P}, s, \chi) \models p & \text { iff } & s \in \pi(p) \\
(\mathscr{P}, s, \chi) \models X \psi & \text { iff } & \text { for } \lambda=\text { out }\left(s, F^{\chi}\right),(\mathscr{P}, \lambda(1), \chi) \models \psi \\
(\mathscr{P}, s, \chi) \models \psi U \psi^{\prime} & \text { iff } & \text { for } \lambda=\operatorname{out}\left(s, F^{\chi}\right) \text { there is } k \geq 0 \text { s.t. }(\mathscr{P}, \lambda(k), \chi) \models \psi^{\prime} \\
& & \text { and } 0 \leq j<k \text { implies }(\mathscr{P}, \lambda(j), \chi) \models \psi \\
(\mathscr{P}, s, \chi) \models K_{i} \psi & \text { iff } & \text { for all } \in \in S, s \sim_{i} s^{\prime} \text { implies }\left(\mathscr{P}, s^{\prime}, \chi\right) \models \psi \\
(\mathscr{P}, s, \chi) \models \exists x_{i} \psi & \text { iff } & \text { there exists an i-strategy } F_{i} \text { s.t. }\left(\mathscr{P}, s, \chi_{F_{i}}^{i}\right) \models \psi
\end{array}
$$

An ESL formula $\varphi$ is satisfied at state $s$, or $(\mathscr{P}, s) \models \varphi$, if $(\mathscr{P}, s, \chi) \models \varphi$ for all assignments $\chi$; $\varphi$ is true in $\mathscr{P}$, or $\mathscr{P} \models \varphi$, if $\left(\mathscr{P}, s_{0}\right) \models \varphi$. The satisfaction of formulas is independent from bound variables, that is, $\chi(f r(\phi))=\chi^{\prime}(f r(\phi))$ implies that $(\mathscr{P}, s, \chi) \models \phi$ iff $\left(\mathscr{P}, s, \chi^{\prime}\right) \models \phi$. In particular, the satisfaction of sentences is independent from assignments.

We can now state the model checking problem for ESL.

Definition 6 (Model Checking Problem) Given an ECGM $\mathscr{P}$ and an ESL formula $\phi$, determine whether there exists an assignment $\chi$ s.t. $\left(\mathscr{P}, s_{0}, \chi\right) \models \phi$.

Notice that, if $y_{1}, \ldots, y_{m}$ is an enumeration of $\operatorname{fr}(\phi)$, then the model checking problem amounts to check whether $\mathscr{P} \models \exists y_{1}, \ldots, \exists y_{m} \phi$, where $\exists y_{1}, \ldots, \exists y_{m} \phi$ is a sentence.

Hereafter we illustrate the formal machinery introduced thus far with a toy example.

Example. We introduce a turn-based ECGM with two agents, $A$ and $B$. First, $A$ secretly chooses between 0 and 1 . Then, at the successive stage, $B$ also chooses between 0 and 1 . The game is won by agent $A$ if the values provided by the two agents coincide, otherwise $B$ wins. We formally describe this toy game starting with agents $A$ and $B$. Specifically, $A$ is the tuple $\left\langle L_{A}, A c t_{A}, P r_{A}\right\rangle$, where (i) $L_{A}=\left\{\varepsilon_{A}, 0,1\right\}$; (ii) $A c t_{A}=\{\operatorname{set}(0)$, set (1), skip $\}$; and (iii) $\operatorname{Pr}_{A}\left(\varepsilon_{A}\right)=\{\operatorname{set}(0)$, set (1) $\}$ and $\operatorname{Pr}_{A}(0)=$ $\operatorname{Pr}_{A}(1)=\{$ skip $\}$. Further, agent $B$ is defined as the tuple $\left\langle L_{B}, A c t_{B}, \operatorname{Pr}_{B}\right\rangle$, where $L_{B}=\left\{\varepsilon_{B}, \lambda, 0,1\right\} ; A c t_{B}=$ $\{$ wait, set $(0)$, set (1), skip $\} ; \operatorname{Pr}_{B}\left(\varepsilon_{B}\right)=\{$ wait $\}, \operatorname{Pr}_{B}(\lambda)=\{\operatorname{set}(0), \operatorname{set}(1)\}$ and $\operatorname{Pr}_{B}(0)=\operatorname{Pr}_{B}(1)=\{$ skip $\}$. The intuitive meaning of local states, actions and protocol functions is clear. Also, we consider the set $A P=\left\{\operatorname{win}_{A}\right.$, win $\left._{B}\right\}$ of atomic propositions, which intuitively express that agent $A$ (resp. $B$ ) has won the game. We now introduce the ECGM $\mathscr{Q}$, corresponding to our toy game, as the tuple $\left\langle A g, s_{0}, \tau, \pi\right\rangle$, where (i) $s_{0}=\left(\varepsilon_{A}, \varepsilon_{B}\right)$; (ii) the transition function $\tau$ is given as follows for $i, j \in\{0,1\}$ :

- $\tau\left(\left(\varepsilon_{A}, \varepsilon_{B}\right),(\operatorname{set}(i)\right.$, wait $\left.)\right)=(i, \lambda)$

- $\tau((i, \lambda),(\operatorname{skip}, \operatorname{set}(j)))=(i, j)$

- $\tau((i, j),($ skip, skip $))=\left(\varepsilon_{A}, \varepsilon_{B}\right)$

and $($ iii $) \pi\left(\operatorname{win}_{A}\right)=\{(0,0),(1,1)\}, \pi\left(\operatorname{win}_{B}\right)=\{(1,0),(0,1)\}$. Notice that we suppose that our toy game, represented in Fig. 1 , is non-terminating.

Now, we check whether the following ESL specifications hold in the ECGM $\mathscr{Q}$.

$$
\begin{aligned}
\mathscr{Q} & =\forall x_{A} X K_{B} \exists y_{B} X \operatorname{win}_{B} \\
\mathscr{Q} & \models \forall x_{A} X \exists y_{B} K_{B} X \operatorname{win}_{B} \\
\mathscr{Q} & =\forall x_{A} X K_{B} K_{A} \exists y_{B} X \operatorname{win}_{A} \\
\mathscr{Q} & =\forall x_{A} X K_{B} \exists y_{B} K_{A} X \operatorname{win}_{A}
\end{aligned}
$$




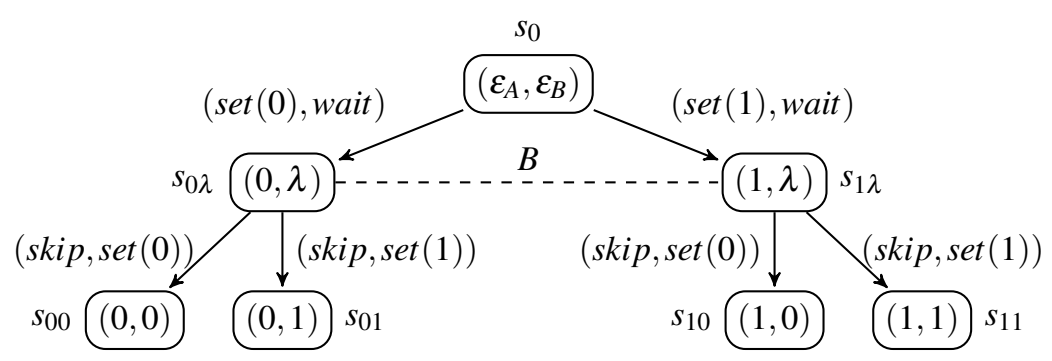

Figure 1: the ECGM Q. Transitions from $s_{00}, s_{01}, s_{10}$, and $s_{11}$ to $s_{0}$ are omitted.

Intuitively, (1) expresses the fact that at the beginning of the game, independently from agent $A$ 's move, at the next step agent $B$ knows that there exists a move by which she can enforce her victory. That is, if agent $A$ chose 0 (resp. 1), then $B$ can choose 1 (resp. 0 ). However, $B$ only knows that there exists a move, but she is not able to point it out. In fact, (2) does not hold, as $B$ does not know which specific move $A$ chose, so she is not capable of distinguishing states $s_{0 \lambda}$ and $s_{1 \lambda}$. Moreover, by (3) $B$ knows that $A$ knows that there exists a move by which $B$ can let $A$ win. Also, by (4) this move is known to $A$, as it is the $B$-move matching $A$ 's move.

Indeed, in ESL it is possible to express the difference between de re and de dicto knowledge of strategies. One of the first contributions to tackle this issue formally is [14]. Formula (1) expresses agent $B$ 's de dicto knowledge of strategy $y_{B}$; while (2) asserts de re knowledge of the same strategy. Similarly, in (3) agent $A$ has de re knowledge of strategy $y_{B}$; while (4) states that agent $A$ knows the same strategy de dicto. The de relde dicto distinction is of utmost importance as, as shown above, having a de dicto knowledge of a strategy does not guarantee that an agent is actually capable of performing the associated sequence of actions. Ideally, in order to have an effective strategy, agents must know it de re.

\section{Model Checking ESL}

In this section we consider the complexity of the model checking problem for ESL. In Section 4.1 and 4.2 we provide the lower and upper bound respectively. For reasons of space, we do not provide full proofs, but only give the most important partial results. We refer to [3] for detailed definitions and complete proofs.

For an ESL formula $\phi$ we define alt $(\phi)$ as the maximum number of alternations of quantifiers $\exists$ and $\forall$ in $\phi$. Then, ESL[ $k$-alt] is the set of ESL formulas $\phi$ with alt $(\phi)$ equal to or less than $k$.

\subsection{Lower Bound}

In this section we prove that model checking ESL formulas is non-elementary-hard. Specifically, we show that for ESL formulas with maximum alternation $k$ the model checking problem is $k$-EXPSPACEhard. The proof strategy is similar to [17], namely, we reduce the satisfiability problem for quantified propositional temporal logic (QPTL) to ESL model checking. However, the reduction applied is different, as ESL does not contain the bind operator used in [17].

We first state that the satisfiability problem for QPTL sentences built on a finite set $A P=\left\{p_{0}, \ldots, p_{n}\right\}$ of atomic propositions can be reduced to model checking ESL sentences on a ECGM $\mathscr{Q}$ of fixed size on $|A P|$, albeit exponential. 
Lemma 1 (QPTL Reduction) Let $A P=\left\{p_{0}, \ldots, p_{n}\right\}$ be a finite set of atomic propositions. There exists an ECGM $\mathscr{Q}$ on AP s.t. for every QPTL[k-alt] sentence $\phi$ on AP, there exists an ESL[k-alt] sentence $\bar{\phi}$ s.t. $\phi$ is satisfiable iff $\mathscr{Q}=\bar{\phi}$.

By this result and the fact that the satisfiability problem for QPTL[k-alt] is $k$-EXPSPACE-hard [17], we can derive the lower bound for model checking ESL[ $k$-alt].

Theorem 2 (Hardness) The model checking problem for ESL[k-alt] is k-EXPSPACE-hard.

In particular, it follows that ESL model checking is non-elementary-hard.

\subsection{Upper Bound}

In this section we extend to Epistemic Strategy Logic the model checking procedure for SL in [17], which is based on alternating tree automata (ATA) [18]. We state the following result, which extends Lemma 5.6 in [17].

Lemma 3 Let $\mathscr{P}$ be an ECGM and $\phi$ an ESL formula. Then, there exists an alternating tree automaton $\mathscr{A}_{\mathscr{P}}^{\phi}$ s.t. for every state $s \in S$ and assignment $\chi$, we have that $(\mathscr{P}, s, \chi) \models \phi$ iff the assignment-state encoding $\mathscr{T}_{s}^{\chi}$ belongs to the language $\mathscr{L}\left(\mathscr{A}_{\mathscr{P}}^{\phi}\right)$.

The following result corresponds to Theorem 5.4 in [17].

Theorem 4 (ATA Direction Projection) Let $\mathscr{A}_{\mathscr{P}}^{\phi}$ be the ATA in Lemma 3 and $s \in S$ a distinguished state. Then, there exists a non-deterministic ATA $\mathscr{N}_{\mathscr{P}, s}^{\phi}$ s.t. for all Act $t_{f r}(\phi)$-labelled $\Delta$-tree $\mathscr{T}=\langle T, V\rangle$, we have that $\mathscr{T} \in \mathscr{L}\left(\mathscr{N}_{\mathscr{P} . s}^{\phi}\right)$ iff $\mathscr{T}^{\prime} \in \mathscr{L}\left(\mathscr{A}_{\mathscr{P}}^{\phi}\right)$, where $\mathscr{T}^{\prime}$ is the $\left(\right.$ Act $\left._{f r(\phi)} \times S\right)$-labelled $\Delta$-tree $\left\langle T, V^{\prime}\right\rangle$ s.t. $V^{\prime}(x)=\left(V(x)\right.$, last $\left.\left(\kappa_{s \cdot x}\right)\right)$.

Then, by using Lemma 3 and Theorem 4 we can state the following result.

Theorem 5 Let $\mathscr{P}$ be an ECGM, s a state in $\mathscr{P}, \chi$ an assignment, and $\phi$ an ESL formula. The nondeterministic ATA $\mathscr{N}_{\mathscr{P}, s}^{\phi}$ in Theorem 4 is such that $(\mathscr{P}, s, \chi) \models \phi$ iff $\mathscr{L}\left(\mathscr{N}_{\mathscr{P}, s}^{\phi}\right) \neq \emptyset$.

We can finally state the following extension to Theorem 5.8 in [17], which follows from the fact that the non-emptyness problem for alternating tree automata is non-elementary in the size of the formula.

Theorem 6 (Completeness) The model checking problem for ESL is PTIME-complete w.r.t. the size of the model and NON-ELEMENTARYTIME w.r.t. the size of the formula.

We remark that Theorem 6 can be used to show that the model checking problem for the nestedgoal fragment ESL[NG] is PTIME-complete w.r.t. the size of the model and $(k+1)$-EXPTIME w.r.t. the maximum alternation $k$ of a formula. We conclude that the complexity of model checking ESL is not worse than the corresponding problem for the Strategy Logic in [17].

\section{Conclusions}

In this paper we introduced Epistemic Strategy Logic, an extension of Strategy Logic [17] with modalities for individual knowledge. We provided this specification language with a semantics in terms of epistemic concurrent game models (ECGM), and analysed the corresponding model checking problem. A number of developments for the proposed framework are possible. Firstly, the model checking problem for the nested-goal, boolean-goal, and one-goal fragment of SL has lower complexity. It is likely that similar results hold also for the corresponding fragments of ESL. Secondly, we can extend ESL with modalities for group knowledge, such as common and distributed knowledge. Thirdly, we can consider various assumptions on ECGM, for instance perfect recall, no learning, and synchronicity. The latter two extensions, while enhancing the expressive power of the logic, are also likely to increase the complexity of the model checking and satisfiability problems. 


\section{References}

[1] Thomas Agotnes, Valentin Goranko \& Wojciech Jamroga (2007): Alternating-time Temporal Logics with Irrevocable Strategies. In: Proceedings of the 11th Conference on Theoretical Aspects of Rationality and Knowledge, TARK '07, ACM, New York, NY, USA, pp. 15-24, doi:10.1145/1324249.1324256.

[2] Rajeev Alur, Thomas A. Henzinger \& Orna Kupferman (2002): Alternating-time temporal logic. J. ACM 49(5), pp. 672-713, doi:10.1145/585265.585270.

[3] Francesco Belardinelli (2014): Reasoning about Knowledge and Strategies: Epistemic Strategy Logic. Technical Report, Universit d'Evry, Laboratoire IBISC. Available at https://www.ibisc.univ-evry.fr/ $\sim$ belardinelli/Documents/sr2014.pdf.

[4] Nils Bulling, Jurgen Dix \& Wojciech Jamroga (2010): Model Checking Logics of Strategic Ability: Complexity*. In Mehdi Dastani, Koen V. Hindriks \& John-Jules Charles Meyer, editors: Specification and Verification of Multi-agent Systems, Springer US, pp. 125-159, doi:10.1007/978-1-4419-6984-2.

[5] Krishnendu Chatterjee, Thomas A. Henzinger \& Nir Piterman (2010): Strategy logic. Inf. Comput. 208(6), pp. 677-693, doi:10.1016/j.ic.2009.07.004.

[6] Ronald Fagin, Joseph Y. Halpern, Yoram Moses \& Moshe Y. Vardi (1995): Reasoning About Knowledge. The MIT Press.

[7] Peter Gammie \& Ron van der Meyden (2004): MCK: Model Checking the Logic of Knowledge. In Rajeev Alur \& Doron Peled, editors: CAV, Lecture Notes in Computer Science 3114, Springer, pp. 479-483, doi:10.1007/978-3-540-27813-9_41.

[8] Valentin Goranko \& Wojciech Jamroga (2004): Comparing Semantics of Logics for Multi-Agent Systems. Synthese 139(2), pp. 241-280, doi:10.1023/B:SYNT.0000024915.66183.d1

[9] Joseph Y. Halpern \& Moshe Y. Vardi (1986): The Complexity of Reasoning about Knowledge and Time: Extended Abstract. In Juris Hartmanis, editor: STOC, ACM, pp. 304-315, doi 10.1145/12130.12161.

[10] Joseph Y. Halpern \& Moshe Y. Vardi (1989): The Complexity of Reasoning about Knowledge and Time. I. Lower Bounds. J. Comput. Syst. Sci. 38(1), pp. 195-237, doi:10.1016/0022-0000(89)90039-1.

[11] Wiebe van der Hoek \& Michael Wooldridge (2003): Cooperation, Knowledge, and Time: Alternating-time Temporal Epistemic Logic and its Applications. Studia Logica 75(1), pp. 125-157, doi $10.1023 / \mathrm{A}: 1026185103185$.

[12] Wojciech Jamroga (2004): Some Remarks on Alternating Temporal Epistemic Logic. In: Proceedings of Formal Approaches to Multi-Agent Systems (FAMAS 2003), pp. 133-140.

[13] Wojciech Jamroga \& Thomas Ågotnes (2007): Constructive knowledge: what agents can achieve under imperfect information. Journal of Applied Non-Classical Logics 17(4), pp. 423-475, doi:10.3166/jancl.17.423475.

[14] Wojciech Jamroga \& Wiebe van der Hoek (2004): Agents that Know How to Play. Fundam. Inform. 63(2-3), pp. 185-219. Available at http://iospress.metapress.com/content/xh738axb47d8rchf/.

[15] Geert Jonker (2003): Feasible strategies in Alternating-time Temporal Epistemic Logic. Master's thesis, University of Utrecht.

[16] Alessio Lomuscio, Hongyang Qu \& Franco Raimondi (2009): MCMAS: A Model Checker for the Verification of Multi-Agent Systems. In A. Bouajjani \& O. Maler, editors: CAV, Lecture Notes in Computer Science 5643, Springer, pp. 682-688, doi:10.1007/978-3-642-02658-4_55.

[17] Fabio Mogavero, Aniello Murano, Giuseppe Perelli \& Moshe Y. Vardi (2011): Reasoning About Strategies. CoRR abs/1112.6275. Available at http://arxiv.org/abs/1112.6275.

[18] David E. Muller \& Paul E. Schupp (1987): Alternating Automata on Infinite Trees. Theor. Comput. Sci. 54, pp. 267-276, doi:10.1016/0304-3975(87)90133-2

[19] Marc Pauly (2002): A Modal Logic for Coalitional Power in Games. J. Log. Comput. 12(1), pp. 149-166, doi $10.1093 / \log \operatorname{com} / 12.1 .149$. 